\title{
Development of the Laboratory Work: "Modeling of a Mobile Robot on Mecanum Wheels Kinematics"
}

\author{
Daniil S. Alhanov ${ }^{1}$ and Vasily I. Rubtsov ${ }^{1 *}$ \\ ${ }^{1}$ Bauman Moscow State Technical University, 2nd Baumanskaya str., 5/1, 105005, Moscow, Russia
}

\begin{abstract}
The basis of any mobile robot is the chassis of a structure. The chassis is a set of parts that provide the mechanical energy transfer from the engines to the engine active elements, combined with the drives in a functional structural subsystem. The chassis and the applied propulsion determine both the mobile robot design appearance and its functional capabilities, the main of which is the ability to move in given conditions. Omnicycle movement is used in modern robotics to get additional maneuverability of the device in combination with increasing the efficiency of using a limited level of energy. Such systems allow for omnidirectional movement by applying an individual torque to the drive of each wheel. The main advantage of the kinematics of such robots is the fact that the complex movement of the entire system can easily be divided into simple rotational and translational movements. This article discusses the design features and the mecanum wheel kinematics. We solve direct and inverse kinematics problems for a four-wheeled mobile robot with this type of propellers on a flat surface. Based on the got dependencies, we built a mathematical model in the Matlab Simulink environment using an additional Mobile Robotics Simulation Toolbox package. Using the obtained model, we developed the simplest trajectory elements and synthesized algorithms for managing the mobile platform. Also, we analyzed the results got during a series of computer tests.
\end{abstract}

\section{Introduction}

The basis of any mobile robot is the chassis of a structure. The chassis is a set of parts that provide the transfer of mechanical energy from the engines to the active ele-ments of the engine, united with the drives in a functional structural sub-system. The chassis and the applied engine largely determine both the design appearance of the mobile robot and its functional capabilities, the main of which is the ability to move in a non - deterministic environment and adaptability to complex driving conditions [1].

Omnicycle movement is used in modern robotics to get additional maneuverability of the device in combination with increasing the efficiency of using a limited level of energy. However, these advantages are inextricably linked either to the inevitably increasing

\footnotetext{
*Corresponding author: rubtsov@mail.ru
} 
complexity of the mechanical design of propellers or to the complexity of the control algorithm structure [2].

Using roller wheels allows you to find a compromise and simplify the structure of the control program of the mobile robot. Such systems allow for omnidirectional movement by applying individual torque to the drive of each wheel. The resulting complex movement of the entire system can easily be divided into simple rotational and translational movements, which simplifies the algorithms for analyzing the flow state and controlling the mobile robot [3].

The advantage of using roller wheels is the ability to turn in place with minimal friction and low torque, combined with the absence of significant ground defor-mation [4]. Such a wheel platform is controlled by individually controlling the speed and direction of rotation of each drive [5]. The contact forces from each wheel even-tually form the resultant force, which is the robot's motion vector. Because the above-described forces of contact interaction depend on the spatial orientation of the wheel and the rollers on it, it is necessary to ensure the preservation of the engine position relative to the support surface [6]. To perform a maneuver, the drive of such a wheel need not to develop a large moment, but the weight and cost of the final design are significantly higher compared to the chassis on conventional engines [7].

\section{Methods}

\subsection{Mecanum Wheel Design Analysis}

Most often, the following version of the design of the roller wheel is used in practice - the so-called mecanum wheel. In such a wheel, rollers are fixed on the periphery (outer rim), so that the wheel rests on the bearing surface only with one of the rollers. Each roller rotates freely around an axis that is stationary relative to the disk plane, and the wheel can roll in a straight line that is a fixed angle with the wheel plane. For mecanum wheels, the roller axis is fixed at an angle of $\gamma=45^{\circ}$ to the wheel plane. There are two versions of the design of such roller wheels (see Fig. 1).

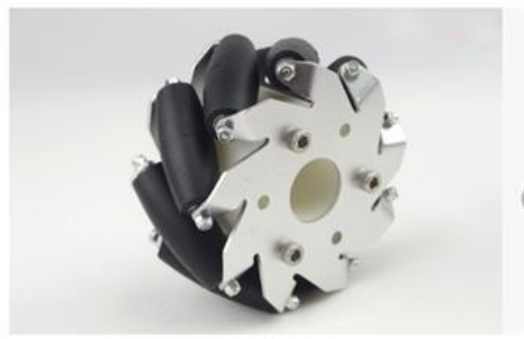

a)

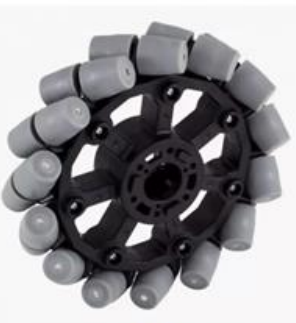

b)

Fig. 1. Mecanum wheel design options.

The difference in the design of mecanum wheels is reflected in their characteristics. The wheel presented in option a) is capable of handling heavy loads and is more stable than the wheel of option b), but the possibility of such a wheel on an uneven surface is lower since there is a risk of hitting the metal attachment on an obstacle during lateral movement. However, kinematic dependencies are not affected by design differences.

At the initial design stage, wheels are defined with the number of the rollers on the wheel and their geometric parameters. The peculiar shape of the rollers forms a circular 
silhouette of the wheel, ensuring smooth running in a straight line, despite the discreteness of contact with the surface. The curve of the forming roller must satisfy equation [8]:

$$
y=r-k x^{2}
$$

The location of the rollers and their minimum number also significantly affect the rolling character of the wheel. The initial data for the design are the roller length $L_{r}$ and the radius of the wheel $R$. The design scheme for calculating the parameters of the elements of the mecanum wheel is simplified in the figure below (see Fig. 2).

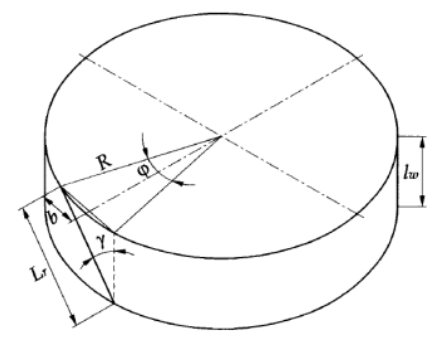

Fig. 2. The wheel design parameter calculation scheme.

Let $\varphi$ be the angle between the lines connecting the geometric center of the wheel and the projection of the roller ends on the disk plane. Then the required number of rollers can be determined from the ratio:

$$
n=\frac{2 \cdot \pi}{\varphi}
$$

If the number of rollers $n$ is defined, we can write an expression for the length of the roller:

$$
L_{r}=2 \cdot R \cdot \frac{\sin \frac{\varphi}{2}}{\sin \gamma}=2 \cdot R \cdot \frac{\sin \frac{\pi}{n}}{\sin \gamma}
$$

At the last stage of calculation, the disk width is determined $L_{w}$ :

$$
L_{w}=L_{r} \cdot \cos \gamma=2 \cdot R \cdot \frac{\sin \frac{\pi}{n}}{\tan \gamma}
$$

In the case of a mecanum wheel, the angle $\gamma=45^{\circ}$ is defined, which simplifies the calculation formula for the roller length:

$$
L_{r}=2 \sqrt{2} \cdot R \cdot \sin \frac{\pi}{n}
$$

The ratio for disk width is simplified in the same way:

$$
L_{w}=2 \cdot R \cdot \sin \frac{\pi}{n}
$$

This article will consider the kinematics of a wheel designed in the research (see Fig. 3). 

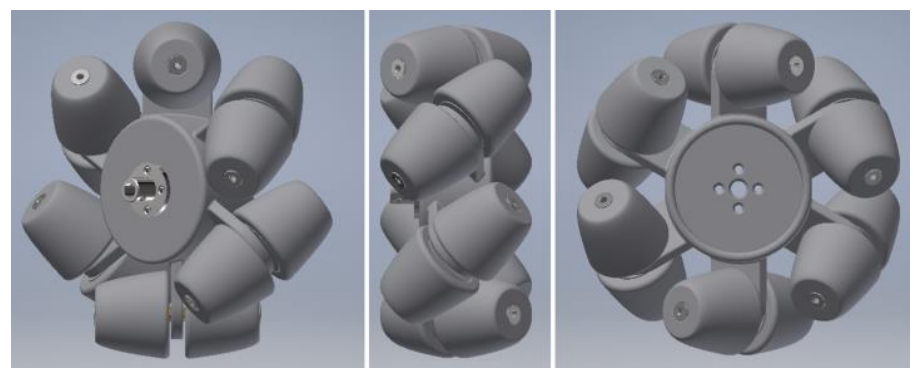

Fig. 3. General view of the mecanum wheel under consideration.

We choose a typical configuration of a mobile robot on a mecanum wheel - a fourwheeled vehicle - as a mobile platform. When installing roller wheels, several requirements must be met. There are left-hand and right-hand versions of mecanum wheels. The difference between them is the location of the rollers - on the left - hand wheels, the rollers are located from the lower right edge to the upper left, and on the right-hand side-from the lower left to the upper right. A four-wheeled mobile robot must be equipped with two lefthand and two right-hand roller wheels [9]. It is recommended to install the wheels so that the axis of rotation of the upper roller points to the center of the platform, which will facilitate system modeling and control algorithms [10]. As a mobile platform, this article considers a simplified model of the mobile robot chassis prototype presented below (see Fig. 4).
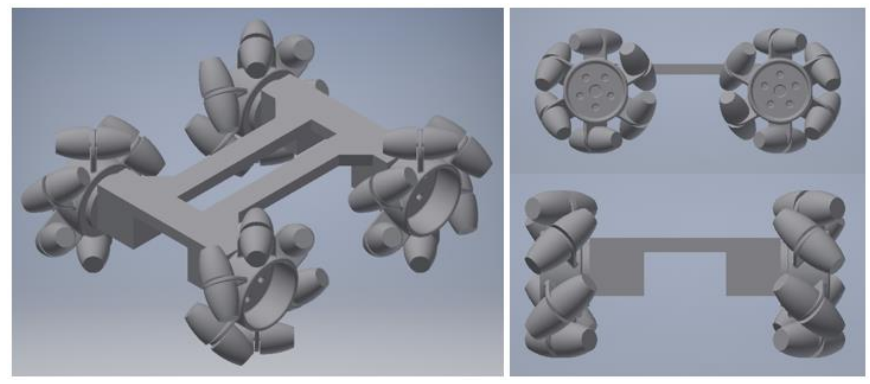

Fig. 4. General view of the mobile platform under consideration.

\subsection{Kinematic Dependencies Analysis}

In Fig. 5 you can see the configuration of the system parameters for an arbitrary robot position relative to a fixed coordinate system associated with the earth. 


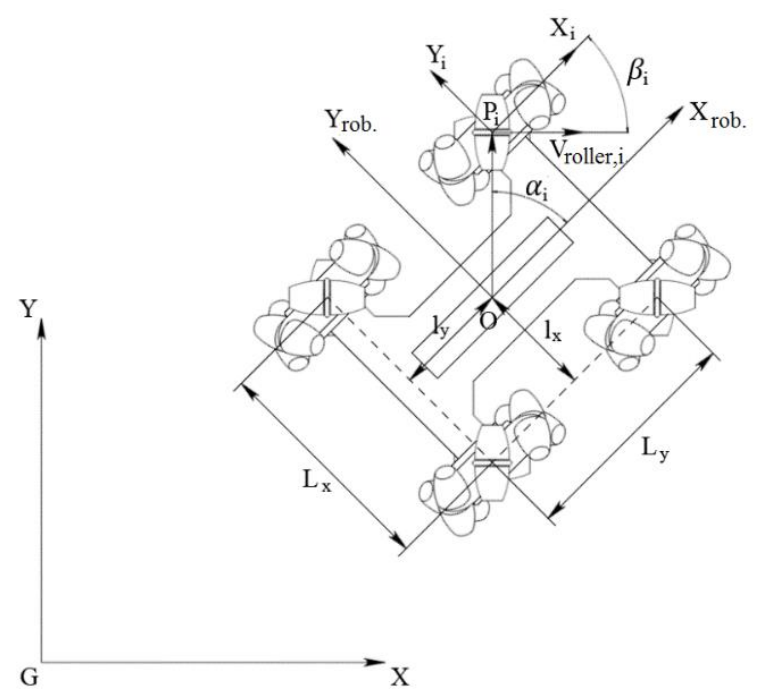

Fig. 5. Mobile robot parameters at any time.

You can see the wheel parameters in the coordinate system associated with the robot's center of mass in Fig. 6.

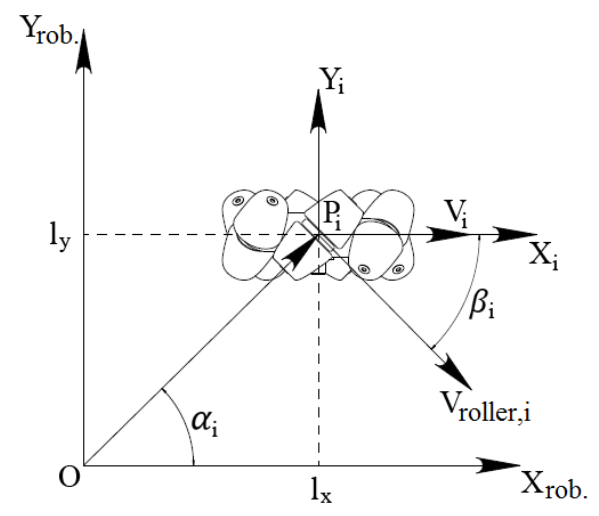

Fig. 6. Caption of the Figure 1. Below the figure. Fig. 6. Wheel parameters in the robot's coordinate system.

We have entered the following notation:

- coordinate system $X G Y$ - the underlying inertial coordinate system;

- coordinate system $X_{\text {rob. }} O Y_{\text {rob. }}$ - coordinate system associated with the robot's center of mass;

- coordinate system $X_{i} P_{i} Y_{i}$ - coordinate system of the $i$-th wheel with the center at a point $P_{i}$ coinciding with the geometric center of the wheel;

- $\quad L_{x}, L_{y}$ - the distance between the geometric centers of the wheels along the width and length of the platform, respectively;

- $l_{x}, l_{y}$ - the distances from the robot center of mass to the axis of the wheels geometric centers;

- $\quad l_{i}-$ the distance between the center of the wheel and the robot center of mass ; 
- $x, y, \theta$ - current coordinates of the robot's center of mass $(x, y)$ in the base coordinate system and the orientation angle of the platform $\theta$ - the angle between the axes $X$ and $X_{\text {rob. }}$;

- $V_{x}, V_{y}-$ the robot linear velocity;

- $\omega_{z}-$ the robot angular velocity;

- $\quad \omega_{i}$ - angular velocity of the $i$-th wheel;

- $\quad V_{i}$ - linear velocity of the $i$-th wheel;

- $\quad V_{\text {roller }, i}$ - linear velocity of the roller on the $i$-th wheel;

- $\quad \overrightarrow{O P_{l}}$ - vector of the distance from the robot's center of mass to the geometric center of the $i$-th wheel;

- $r_{\text {wheel }}-$ wheel radius (distance from the wheel geometric center to the roller geometric center);

- $\quad r_{\text {roller }}$ - roller radius;

- $\alpha_{i}$ - the angle between vector $\overrightarrow{O P_{l}}$ and axis $X_{\text {rob }}$.

- $\quad \beta_{i}$ - the angle between roller linear velocity $V_{\text {roller }, i}$ and axis $X_{i}$.

The system kinematics analysis begins with the consideration of the wheel movement principle on the surface. The wheel rolling can be represented as the sum of translational movement and rotational movement. For an ordinary wheel of a standard design, there are two possible cases of surface movement: non-slip and non-slip movement [11]. In the case of motion without slippage, the statement about the instantaneous center of speeds and zero speed at the point of contact is valid (see Fig. 7).

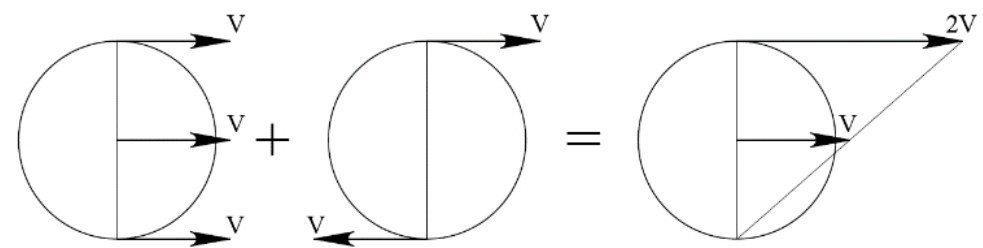

Fig. 7. Diagram of the wheel movement without slipping.

Each of the mecanum wheel rollers can move independently of the disk. Therefore, when moving the disk of the mecanum wheel "slips" on the surface of the rotating rollers. We assumed that the rollers themselves do not slip when they contact the surface. We should also note that in the absence of an angle between the roller and the disk, the wheel would have to be rotated in the opposite direction to the movement. This does not happen because of the presence of an angle and the formation of a kind of stop. This complex movement can be considered as the movement of a standard-design wheel with a slip (see Fig. 8).

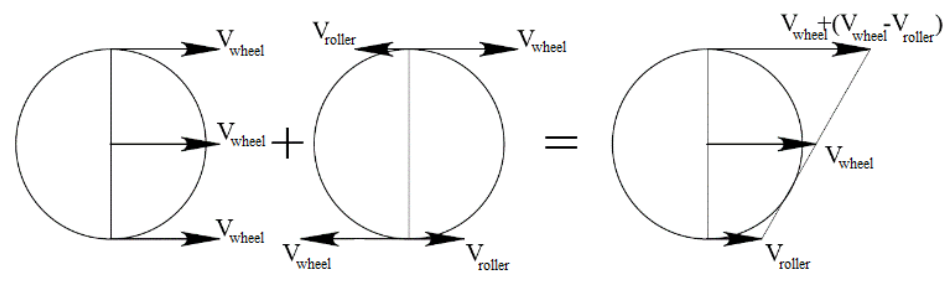

Fig. 8. Diagram of the wheel movement in the presence of slippage. 
Configuration in which the wheel disk contacts the surface through a single roller is convenient to study the wheel movement kinematic dependencies. In this case, the axis from the point of roller attachment on the disk to the disk geometric center is perpendicular to the wheel coordinate system (see Fig. 9).

Since each roller is fixed directly on the wheel disk, continuously moves together with the disk, and is in contact with the surface for a short time, the angular speed of the wheel and the roller are assumed to be equal [12].

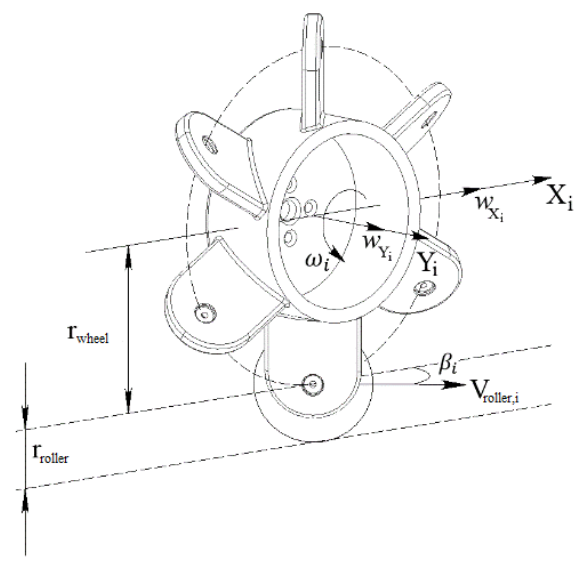

Fig. 9. Diagram of the wheel movement when the roller contacts the surface.

Since we assumed that the roller moves on the surface without slipping, we made the following expression:

$$
V_{\text {roller }, i}=\frac{1}{\cos \beta_{i}} \cdot r_{\text {roller }} \cdot \omega_{i}, i=1 \ldots 4
$$

The wheel disk moves with "slippage", so its velocity will be determined by the sum of two components:

$$
\begin{gathered}
w_{X_{i}}=r_{\text {wheel }} \cdot \omega_{i}, i=1 \ldots 4 \\
V_{X_{i}}=w_{X_{i}}+V_{\text {roller }, i} \cdot \cos \beta_{i}=r_{\text {wheel }} \cdot \omega_{i}+r_{\text {roller }} \cdot \omega_{i}
\end{gathered}
$$

You can found the projection of the linear velocity of the wheel on the $Y_{i}$ axis as:

$$
V_{Y_{i}}=w_{Y_{i}}=V_{\text {roller }, i} \cdot \sin \beta_{i}
$$

The equation for the wheel linear velocity projections in matrix form is written as:

$$
\left[\begin{array}{c}
V_{X_{i}} \\
V_{Y_{i}}
\end{array}\right]=\left[\begin{array}{cc}
r_{\text {wheel }} & \cos \beta_{i} \\
0 & \sin \beta_{i}
\end{array}\right] \cdot\left[\begin{array}{c}
\omega_{i} \\
V_{\text {roller }, i}
\end{array}\right]=T_{P_{i}} \cdot\left[\begin{array}{c}
\omega_{i} \\
V_{\text {roller }, i}
\end{array}\right]
$$

Thus, the matrix $T_{P_{i}}$ in equation (11) is the transition matrix to the coordinate system associated with the wheel geometric center. Since in this case of a four-wheeled mobile robot, there is no angle between the coordinate system associated with the robot center of mass and the coordinate system associated with the wheel geometric center, the velocity of the $i$-th wheel is projected without changes:

$$
\left[\begin{array}{c}
V_{X_{\text {rob } .}} \\
V_{Y_{\text {rob } .}}
\end{array}\right]=\left[\begin{array}{ll}
1 & 0 \\
0 & 1
\end{array}\right] \cdot\left[\begin{array}{c}
V_{X_{i}} \\
V_{Y_{i}}
\end{array}\right]=T_{P_{i}} \cdot\left[\begin{array}{c}
\omega_{i} \\
V_{\text {roller }, i}
\end{array}\right]
$$


Since the movement of the mobile robot occurs in the plane in the presence of both translational and rotational movements, it is also necessary to take into account the contribution of angular velocity to the projection of linear velocities of the center of mass:

$$
\left[\begin{array}{c}
V_{X_{\text {rob }, i}} \\
V_{Y_{\text {rob } i}}
\end{array}\right]=\left[\begin{array}{ccc}
1 & 0 & -l_{y} \\
0 & 1 & l_{x}
\end{array}\right] \cdot\left[\begin{array}{c}
V_{x} \\
V_{y} \\
\omega_{z}
\end{array}\right]=T_{O} \cdot\left[\begin{array}{c}
V_{x} \\
V_{y} \\
\omega_{z}
\end{array}\right]
$$

In equation (13), the $T_{O}$ matrix is the transition matrix from the global fixed coordinate system to the coordinate system associated with the mobile robot center of mass. From equations (11) and (12), a model can be obtained for calculating the inverse kinematics of a mobile robot:

$$
\left[\begin{array}{cc}
r_{\text {wheel }} & \cos \beta_{i} \\
0 & \sin \beta_{i}
\end{array}\right] \cdot\left[\begin{array}{ll}
1 & 0 \\
0 & 1
\end{array}\right] \cdot\left[\begin{array}{c}
\omega_{i} \\
V_{\text {roller }, i}
\end{array}\right]=\left[\begin{array}{ccc}
1 & 0 & -l_{y} \\
0 & 1 & l_{x}
\end{array}\right] \cdot\left[\begin{array}{c}
V_{x} \\
V_{y} \\
\omega_{z}
\end{array}\right]
$$

Since $r_{\text {wheel }} \neq 0$, the angle between the disk plane and the roller axis lies in the range $0<\left|\beta_{i}\right|<\frac{\pi}{2}$, and the determinant of the matrix $\operatorname{det}\left(T_{P_{i}}\right) \neq 0$, therefore, by analyzing equations (12) and (13), we can get a relationship between the linear velocities of the robot's center of mass (at the point $\mathrm{O}$ ) and the angular velocity of the $i$-th wheel:

$$
\left[\begin{array}{c}
\omega_{i} \\
V_{\text {roller }, i}
\end{array}\right]=\left[\begin{array}{cc}
r_{\text {wheel }} & \cos \beta_{i} \\
0 & \sin \beta_{i}
\end{array}\right]^{-1} \cdot\left[\begin{array}{ccc}
1 & 0 & -l_{y} \\
0 & 1 & l_{x}
\end{array}\right] \cdot\left[\begin{array}{c}
V_{x} \\
V_{y} \\
\omega_{z}
\end{array}\right]
$$

\subsection{Solving Direct and Inverse Kinematics Problems}

Using the direct kinematics problem, the linear and angular velocities of the entire system can be calculated by knowing the angular velocities of the wheels and the corresponding linear velocities of the rollers:

$$
\left[\begin{array}{c}
V_{x} \\
V_{y} \\
\omega_{z}
\end{array}\right]=T_{\text {D.K. }} \cdot\left[\begin{array}{c}
\omega_{i} \\
V_{\text {roller }, i}
\end{array}\right]
$$

The system of inverse kinematics problem is constructed in the same way:

$$
\left[\begin{array}{c}
\omega_{i} \\
V_{\text {roller }, i}
\end{array}\right]=T_{\mathrm{I} . \mathrm{K}} \cdot\left[\begin{array}{c}
V_{x} \\
V_{y} \\
\omega_{z}
\end{array}\right]
$$

where $T_{\mathrm{I} . K .}=T_{P_{i}}{ }^{-1} \cdot T_{O}, T_{D . K .}=\left(T_{I . K .}{ }^{T} \cdot T_{I . K .}\right)^{-1} \cdot T_{I . K .}{ }^{T}$. Than:

$$
T_{\text {I.K. }}=\left[\begin{array}{cc}
r_{\text {wheel }} & \cos \beta_{i} \\
0 & \sin \beta_{i}
\end{array}\right]^{-1} \cdot\left[\begin{array}{ccc}
1 & 0 & -l_{y} \\
0 & 1 & l_{x}
\end{array}\right]
$$

Given that all the wheels of the mobile robot are the same size, $l_{x}=l_{i} \cdot \cos \alpha_{i}$ and $l_{y}=l_{i} \cdot \sin \alpha_{i}$, we can write a transformation matrix for the inverse kinematics problem:

$$
T_{\mathrm{I} . \mathrm{K} .}=\frac{1}{-r_{\text {wheel }}} \cdot\left[\begin{array}{ccc}
\frac{\cos \left(-\beta_{i}\right)}{\sin \left(\beta_{i}\right)} & \frac{\sin \left(-\beta_{i}\right)}{\sin \left(\beta_{i}\right)} & \frac{l_{i} \cdot \sin \left(-\alpha_{i}-\beta_{i}\right)}{\sin \left(\beta_{i}\right)} \\
-\frac{r_{\mathrm{wheel}}}{\sin \left(\beta_{i}\right)} & -\frac{r_{\mathrm{wheel}}}{\sin \left(\beta_{i}\right)} & -\frac{l_{i} \cdot \sin \left(-\alpha_{i}\right) \cdot r_{\text {wheel }}}{\sin \left(\beta_{i}\right)}
\end{array}\right]
$$


Similarly, you can get the matrix of a direct kinematics problem:

$$
T_{\text {D.K. }}=\frac{1}{l_{i}{ }^{2}+1} \cdot\left[\begin{array}{cc}
-\frac{1}{2} \cdot\left(-l_{i}{ }^{2} \cdot \sin \left(2 \alpha_{i}\right)\right) \cdot r_{\text {wheel }} & \frac{1}{2} \cdot l_{i}{ }^{2} \cdot \sin \left(\beta_{i}+2 \alpha_{i}\right)-\frac{1}{2} \cdot \sin \left(-\beta_{i}\right) \cdot l_{i}{ }^{2}-\sin \left(-\beta_{i}\right) \\
-\frac{1}{2} \cdot r_{\text {wheel }} \cdot\left(l_{i}{ }^{2}-l_{i}{ }^{2} \cdot \cos \left(2 \alpha_{i}\right)+2\right) & -\frac{1}{2} \cdot l_{i}{ }^{2} \cdot \cos \left(\beta_{i}+2 \alpha_{i}\right)+\frac{1}{2} \cdot \cos \left(-\beta_{i}\right) \cdot l_{i}{ }^{2}+\cos \left(-\beta_{i}\right) \\
\cos \left(\alpha_{i}\right) \cdot l_{i} \cdot r_{\text {wheel }} & \cos \left(\alpha_{i}+\beta_{i}\right) \cdot l_{i}
\end{array}\right]
$$

Assuming that there is no slippage between the rollers of the mecanum wheels and the movement surface, we can get the final expression for the inverse kinematics problem of the system:

$$
\left[\begin{array}{l}
\omega_{1} \\
\omega_{2} \\
\omega_{3} \\
\omega_{4}
\end{array}\right]=\frac{-1}{r_{\text {wheel }}} \cdot\left[\begin{array}{ccc}
\frac{\cos \left(-\beta_{1}\right)}{\sin \left(\beta_{1}\right)} & \frac{\sin \left(-\beta_{1}\right)}{\sin \left(\beta_{1}\right)} & \frac{l_{1} \cdot \sin \left(-\beta_{1}-\alpha_{1}\right)}{\sin \left(\beta_{1}\right)} \\
\frac{\cos \left(-\beta_{2}\right)}{\sin \left(\beta_{2}\right)} & \frac{\sin \left(-\beta_{2}\right)}{\sin \left(\beta_{2}\right)} & \frac{l_{2} \cdot \sin \left(-\beta_{2}-\alpha_{2}\right)}{\sin \left(\beta_{2}\right)} \\
\frac{\cos \left(-\beta_{3}\right)}{\sin \left(\beta_{3}\right)} & \frac{\sin \left(-\beta_{3}\right)}{\sin \left(\beta_{3}\right)} & \frac{l_{3} \cdot \sin \left(-\beta_{3}-\alpha_{3}\right)}{\sin \left(\beta_{3}\right)} \\
\frac{\cos \left(-\beta_{4}\right)}{\sin \left(\beta_{4}\right)} & \frac{\sin \left(-\beta_{4}\right)}{\sin \left(\beta_{4}\right)} & \frac{l_{4} \cdot \sin \left(-\beta_{4}-\alpha_{4}\right)}{\sin \left(\beta_{4}\right)}
\end{array}\right] \cdot\left[\begin{array}{c}
V_{x} \\
V_{y} \\
\omega_{z}
\end{array}\right]
$$

You are free to use colour illustrations for the online version of the proceedings, but any print version will be printed in black and white unless special arrangements have been made with the conference organiser. Please check whether or not this is the case. If the print version will be black and white only, you should check your figure captions carefully and remove any reference to colour in the illustration and text. In addition, some colour figures will degrade or suffer loss of information when converted to black and white, and this should be considered when preparing them.

\subsection{Kinematic Model Development}

During the mathematical model development, we simplified kinematic dependencies, taking into account the specific values of the design angles of the mobile robot. These parameters are summarized in Table 1. The distance from the mobile robot center of mass to the wheel geometric centers was replaced with the overall dimensions of the platform.

Table 1.

\begin{tabular}{|c|c|c|}
\hline Wheel number & $\boldsymbol{\alpha}_{\boldsymbol{i}}$ & $\boldsymbol{\beta}_{\boldsymbol{i}}$ \\
\hline 1 & $\frac{\pi}{4}$ & $-\frac{\pi}{4}$ \\
\hline 2 & $-\frac{\pi}{4}$ & $\frac{\pi}{4}$ \\
\hline 3 & $\frac{3 \pi}{4}$ & $\frac{\pi}{4}$ \\
\hline 4 & $-\frac{3 \pi}{4}$ & $-\frac{\pi}{4}$ \\
\hline
\end{tabular}

You can see the final simplified calculation scheme with the parameters used for the mathematical model in Fig. 10. 


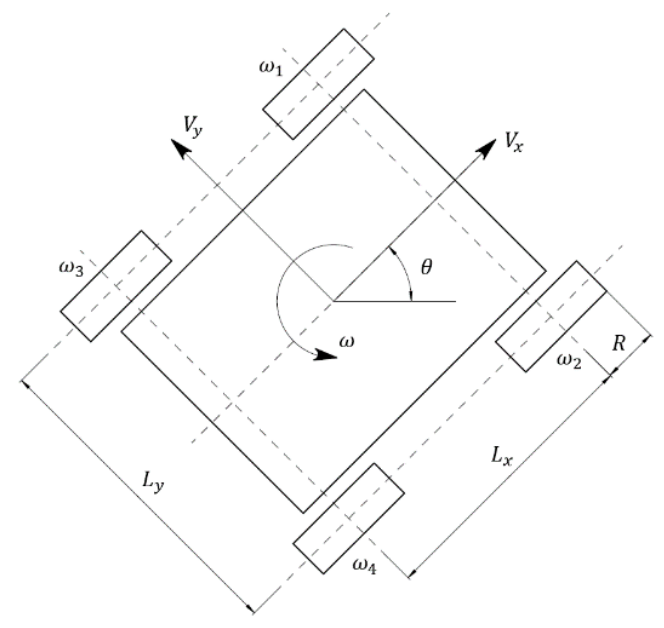

Fig. 10. Calculation scheme of the mathematical model.

Notation on the calculation scheme of the mathematical model:

- $V_{x}, V_{y}-$ mobile robot linear velocity;

- $\omega$ - mobile robot angular velocity;

- $\theta$ - mobile robot orientation (the yaw angle);

- $L_{x}, L_{y}$ - the platform overall dimensions (the distance between the geometric centers of the wheels);

- $\quad \omega_{1} \ldots \omega_{4}-$ mobile robot wheels' angular velocities.

With this configuration, the transformation matrix for the inverse (equation 21) and forward (equation 22) kinematics problems will take the following form:

$$
\begin{gathered}
T_{\text {I.K. }}=\frac{1}{R} \cdot\left[\begin{array}{ccc}
1 & -1 & -\frac{\left(L_{x}+L_{y}\right)}{2} \\
1 & 1 & \frac{\left(L_{x}+L_{y}\right)}{2} \\
1 & 1 & -\frac{\left(L_{x}+L_{y}\right)}{2} \\
1 & -1 & \frac{\left(L_{x}+L_{y}\right)}{2}
\end{array}\right] \\
T_{\text {D.K. }}=\frac{R}{4} \cdot\left[\begin{array}{cccc}
1 & 1 & 1 & 1 \\
-1 & 1 & 1 & -1 \\
-\frac{2}{\left(L_{x}+L_{y}\right)} & \frac{2}{\left(L_{x}+L_{y}\right)} & \frac{2}{\left(L_{x}+L_{y}\right)} & \frac{2}{\left(L_{x}+L_{y}\right)}
\end{array}\right]
\end{gathered}
$$

Following equations (21) and (22), we derived the final formulations of kinematics problems. The direct kinematics problem is defined by the following dependencies:

$$
\left[\begin{array}{l}
V_{x} \\
V_{y} \\
\omega
\end{array}\right]=\frac{R}{4} \cdot\left[\begin{array}{ccccr}
1 & 1 & 1 & 1 \\
-1 & & 1 & 1 & -1 \\
-\frac{2}{\left(L_{x}+L_{y}\right)} & \frac{2}{\left(L_{x}+L_{y}\right)} & \frac{2}{\left(L_{x}+L_{y}\right)} & \frac{2}{\left(L_{x}+L_{y}\right)}
\end{array}\right] \cdot\left[\begin{array}{c}
\omega_{1} \\
\omega_{2} \\
\omega_{3} \\
\omega_{4}
\end{array}\right]
$$

Mobile robot longitudinal velocity:

$$
V_{x}(t)=\left(\omega_{1}+\omega_{2}+\omega_{3}+\omega_{4}\right) \cdot \frac{R}{4}
$$


Mobile robot transverse velocity:

$$
V_{y}(t)=\left(-\omega_{1}+\omega_{2}+\omega_{3}-\omega_{4}\right) \cdot \frac{R}{4}
$$

Mobile robot angular velocity:

$$
\omega(t)=\left(-\omega_{1}+\omega_{2}-\omega_{3}+\omega_{4}\right) \cdot \frac{R}{2\left(L_{x}+L_{y}\right)}
$$

Mobile robot full velocity:

$$
V_{f u l l}=\sqrt{V_{x}^{2}+V_{y}^{2}}
$$

The direction of the mobile robot's motion vector:

$$
\theta=\frac{1}{\tan \left(\frac{V y}{V_{x}}\right)}
$$

The inverse kinematics problem is defined by the following dependencies:

$$
\left[\begin{array}{l}
\omega_{1} \\
\omega_{2} \\
\omega_{3} \\
\omega_{4}
\end{array}\right]=\frac{1}{R} \cdot\left[\begin{array}{ccc}
1 & -1 & -\frac{\left(L_{x}+L_{y}\right)}{2} \\
1 & 1 & \frac{\left(L_{x}+L_{y}\right)}{2} \\
1 & 1 & -\frac{\left(L_{x}+L_{y}\right)}{2} \\
1 & -1 & \frac{\left(L_{x}+L_{y}\right)}{2}
\end{array}\right] \cdot\left[\begin{array}{c}
V_{x} \\
V_{y} \\
\omega
\end{array}\right]
$$

The angular speed of each wheel of the mobile robot:

$$
\left\{\begin{array}{l}
\omega_{1}=\frac{1}{R} \cdot\left(V_{x}-V_{y}-\left(\frac{\left(L_{x}+L_{y}\right)}{2}\right) \cdot \omega\right) \\
\omega_{2}=\frac{1}{R} \cdot\left(V_{x}+V_{y}+\left(\frac{\left(L_{x}+L_{y}\right)}{2}\right) \cdot \omega\right) \\
\omega_{3}=\frac{1}{R} \cdot\left(V_{x}+V_{y}-\left(\frac{\left(L_{x}+L_{y}\right)}{2}\right) \cdot \omega\right) \\
\omega_{4}=\frac{1}{R} \cdot\left(V_{x}-V_{y}+\left(\frac{\left(L_{x}+L_{y}\right)}{2}\right) \cdot \omega\right)
\end{array}\right.
$$

\subsection{Mathematical Model Implementation}

We performed the implementation of the kinematic model provided in 2.4. in the Matlab Simulink software environment using an additional Mobile Robotics Simula-tion Toolbox package. This package provides a Toolkit for the simulation of the mobile robot and the development of control algorithms. In particular, the Mobile Robotics Simulation Toolbox provides $2 \mathrm{D}$ visualization capabilities, working with the leader model and detecting obstacles, etc.

As a result, we developed a debugging mathematical model of a mobile robot on mecanum-wheels, the structural and functional scheme of which is shown in Fig. 11. 


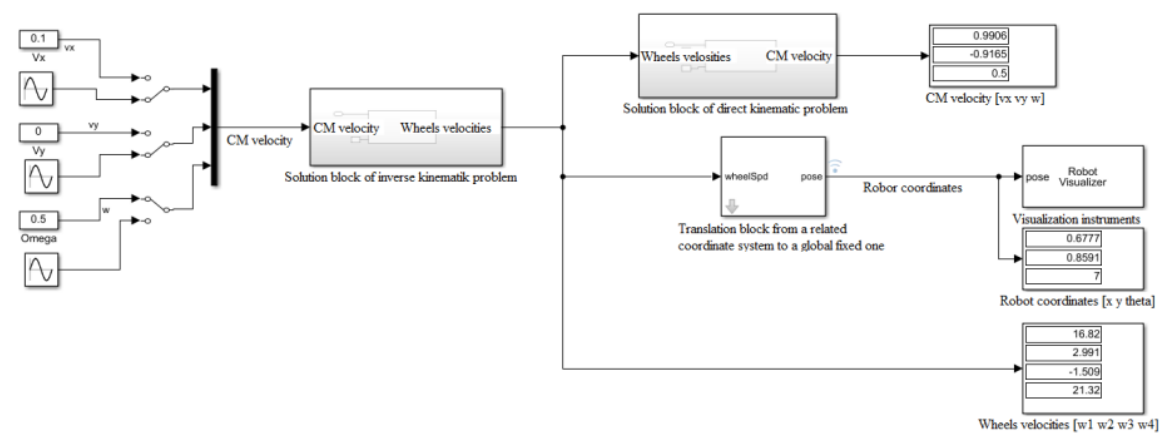

Fig. 11. Debugging mathematical model of robot kinematics

Kinematic dependencies are specified in the blocks for calculating forward and re-verse kinematics problems using Matlab functions. The model uses the wheel radius, geometric parameters of the platform, and the initial orientation of the robot as its initial parameters. Management can be implemented either by using specific parame-ters or by using a certain law. The control signals are either the velocities of each of the mobile robot's wheels or the linear and angular velocities of the center of mass. You can see the result of the mathematical model in Fig. 12. On the left, you can see the result of working with the model when the trajectory is set explicitly (in this case, for a circle), and on the right, you can see the result when working with complex par-ametrically defined trajectory elements.

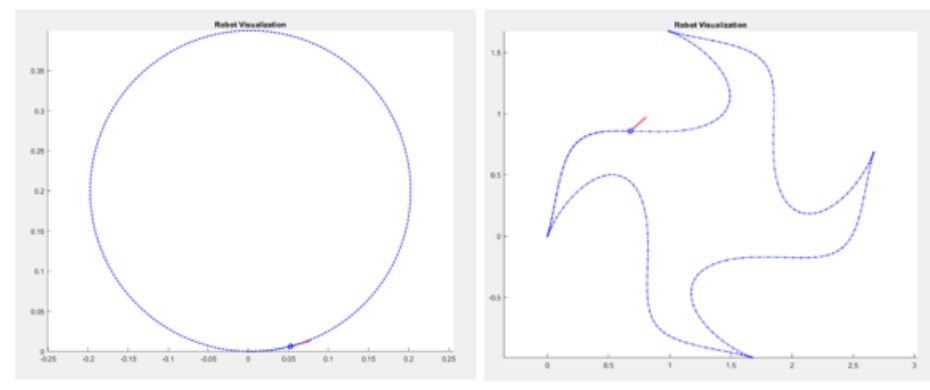

Fig. 12. Modeling results

\section{Conclusion}

As a result of this work, we describe the main features of the mecanum-wheel design and analyzed the kinematic dependencies of the four-wood mobile robot using this type of propellers. We solved direct and inverse kinematics problems for this device configuration and developed a kinematic model of a mobile robot in the Matlab Simulink software environment using an additional Mobile Robotics Simulation Toolbox package. According to the results of 32 computer tests, during which the trajectory elements varied, we accepted the developed mathematical model as work-able.

Based on the results got, we formed a laboratory work on the course "RTS Design" for students of the SM7 Department, which is aimed at:

- familiarization with the design features and design methods of mecanum-wheel;

- learning the functionality of the additional Matlab Mobile Robotics Simulation Toolbox package;

- solution of direct and inverse kinematics problems on the example of a mobile robot on mecanum-wheels; 
- $\quad$ building a mathematical model based on the got dependencies.

\section{References}

1. A.V. Vasil'ev, Principy postroeniya $i$ klassifikaciya shassi mobil'nyh robotov nazemnogo primeneniya i planetohodov [Principles of Construction and Classification of Chassis of Mobile Robots for Ground Use and Planetary Vehicles], Nauchnotekhnicheskie vedomosti SPbGPU. Informatika. Telekommunikacii. Upravlenie [Scientific and Technical Bulletin of SPbGPU. Computer Science. Telecommunications. Management] (2013).

2. E.U. Kolesnichenko, V.E. Pavlovskij, D.A. Gribkov, I.A. Orlov, A.P. Alisejchik, Kinematicheskoe upravlenie dvizheniem shestikolesnogo mekanum-robota [Kinematic Motion Control of a Six-wheeled Mecanum Robot], izdatel'stvo instituta prikladnoj matematiki M.V.Keldysha, RAN [Publishing House of the Keldysh Institute of Applied Mathematics, RAS] (2016).

3. F. Becker, O. Bondarev, I. Zeidis, K. Zimmermann, An Approach to the Kinematics and Dynamics of a Four-wheel Mecanum Vehicle, Special Issue of Scientific Journal of IFToMM “PROBLEMS OF MECHANICS”, № 2(55) (2014).

4. U.G. Martynenko, A.M. Formal'skij, $O$ dvizhenii mobil'nogo robota s rolikonesushchimi kolesami [About the Movement of a Mobile Robot with Roller Wheels], Izvestiya RAN. Teoriya i sistemy upravleniya [Izvestiya RAS. Theory and Control Systems], № 6, pp. 142-149 (2007).

5. Giovanni Indiveri, Swedish wheeled omnidirectional mobile robots: kinematics analysis and control, Dipartimento Ingegneria Innovazione, University of Salento, Lecce, Italy.

6. A.A. Kilin, A.D. Bobykin, Upravlenie telezhkoj s omniwheelmi na ploskosti [Control of a Cart with Omnicycles on a Plane], Nelinejnaya dinamika [Nonlinear Dynamics], vol. 10, № 4, pp. 473-481 (2014).

7. Olaf Diegel, Apama Badve, Glen Bright, Johan Potgieter, Sylvester Tlale, Imoroved Mechanum Wheel Design for Omni-directional Robots, Australian Conference on Robotics and Automation (2002).

8. I. Doroftei, V. Spinu, V. Grosu, Omnidirectional Mobile Robot - Design and Implementation, Bioinspiration and Robotics: Walking and Climbing Robots (2007).

9. R. P. A. van Haendel, Design of an omnidirectional universal mobile platform, Eindhoven University of Technology (2005).

10. Xiang Li, Andreas Zell, Motion Control of an Omnidirectional Mobile Robot, Wilhelm-Schickard-Institute, Departmant of Computer Architecture, University of Tubingen (2012).

11. Hamid Taheri, Bing Qiao, Nurallah Ghaeminezhad, Kinematic Model of a Four Mecanum Wheeled Mobile Robot, International Journal of Computer Applications (0975 - 8887), vol. 113, No. 3.

12. D.U. Motorina, Algoritm postroeniya zapazdyvayushchego upravleniya dlya mobil'nogo kolesnogo robota pri uchete effekta proskal'zyvaniya koles [Algorithm for Constructing a Delayed Control for a Mobile Wheeled Robot with the Account of the Wheel Slip Effect], Matematicheskoe mode-lirovanie, chislennye metody i kompleksy program. Avtomatizaciya processov upravleniya [Mathematical Modeling, Numerical Methods and Software Complexes. Automation of Management Processes], № 3 (25) (2011). 